\title{
La transdisciplinariedad como factor de cambio en el quehacer educativo moderno, a partir de las posturas epistémicas de Edgar Morin y Basarab Nicolescu
}

\author{
José Andrés Herrera Rey ${ }^{1}$ \\ Docente Investigador \\ Universidad Estatal a Distancia, Costa Rica \\ Email: jherrerar@uned.ac.cr \\ ORCID: https://orcid.org/0000-0002-9846-4449
}

Fecha de recepción: 01-11-2020

Fecha de aceptación: 25-02-2021

\section{Resumen}

En este ensayo se realiza un acercamiento al concepto de la transdisciplinariedad manejado por los autores escogidos, en este caso concreto: Edgar Morin y Basarab Nicolescu. Se hace un esfuerzo por definir un concepto general de lo que la transdisciplinariedad significa, rescatando su importancia para sobrellevar los múltiples retos que el mundo actual implica a todos aquellos que ejercen la docencia, así como al estudiantado. Igualmente, se explora en este ensayo una comparación (no exhaustiva), a partir de la identificación de puntos coincidentes y divergentes entre ambos autores, acerca de la temática en análisis, así como de la influencia que consideran que ha ejercido y ejerce esta práctica en los procesos de formación y exigencia académica. Por último, se hace un breve recuento de los posibles impactos de ello, en las experiencias de aprendizaje y su pertinencia en la cotidianidad del proceso educativo.

Palabras clave: Transdisciplinariedad, formación educativa, nueva racionalidad, docentes, discentes, cambios educativos.

\begin{abstract}
In this essay an approach is made to the concept of transdisciplinarity managed by the chosen authors: Edgar Morin and Basarab Nicolescu. An effort is made to define a general concept of what transdisciplinarity means, rescuing its importance to cope with the multiple challenges that the current world involves to those who practice teaching, as well as the student body. Likewise, a comparison (not exhaustive) is explored in this essay, based on the identification of coincident and divergent points between both authors, about the subject under analysis, as well as the influence that they consider that this practice has exerted and exerts on the training processes and academic demands. Finally, a brief account is made of the possible impacts of this, in the learning experiences and its relevance in the daily life of the educational process.
\end{abstract}

Key words: Transdisciplinarity, educational training, new rationality, teachers, learners, educational changes.

1. Sociólogo y educador. Máster en Sociología por la Universidad de Costa Rica. Docente e investigador de la Cátedra de Sociología en la Escuela de Ciencias Sociales y Humanidades de la Universidad Estatal a Distancia de Costa Rica. Doctorando en Educación en la misma casa de estudios. 


\section{Introducción}

Existe una discusión latente respecto de la transdisciplinariedad en la educación superior, tanto en la esfera de los académicos como en el ámbito social. Esto debido a que existe un cúmulo de experiencias, las cuales brindan razones suficientes para hacer una pausa y analizar seriamente el rol, la naturaleza y el alcance que tiene dicho concepto en los actuales espacios de formación académica; así como para reflexionar sobre los efectos que ello tiene sobre el sistema educativo y las implicaciones que devienen en los propios actores involucrados en los distintos procesos y espacios.

Si bien en el mundo se han dado iniciativas que buscan educar a las personas, lo cierto es que no se tiene un análisis unívocamente claro a propósito del alcance y efectividad que dichas políticas tienen en el ejercicio propio de sus funciones. Aunado a ello, si se considera que varios intelectuales, como Morin (1998), Enwistle (2011), Leff (2011) y García (2016), abogan por una racionalidad que posibilite pensar la unidad de los saberes fragmentados en disciplinas, de cara a una mejor comprensión de la realidad del ser humano, resulta cada vez más importante propiciar un modelo de aprendizaje que fomente estructuras o métodos concurrentes entre los distintos saberes, trascendiendo sus propias fronteras.

Acercándose a lo que algunos autores llaman la "globalización de las disciplinas" y aplicado a la esfera del campo personal (Torres, 1998; Teubal, 2008; Santos, 2000, citado en Sobarzo,
2001; Escudero, 2011; Estrada, 2018), se cae en cuenta de la necesidad de dotar de herramientas necesarias y suficientes a los sujetos sociales, con el fin de que puedan adquirir y desarrollar, ulteriormente, habilidades generales y formas de actuar para desenvolverse en el mundo. Fedorov (2008), por ejemplo, señala que parte de esas destrezas y habilidades necesarias que enfrentan las naciones en la actualidad se encuentran muy ligadas a un proceso paulatino, pero sostenido, de un nuevo paradigma, donde la transdisciplinariedad ocupe un rol estelar. Para que, con ello, se consiga en el mediano plazo una población capaz de pensar de forma crítica acerca de la manera en que se decide y se actúa sobre muchas situaciones en el entorno cotidiano.

En suma, con este ensayo se pretende propiciar una reflexión breve sobre la transdisciplinariedad como necesidad real en los sistemas educativos. Se toma como base para reflexionar las propuestas epistémicas de Edgar Morin (pensamiento complejo) y de Basarab Nicolescu (conocimiento transdisciplinario).

\section{Objetivo general}

Analizar las posturas epistémicas de los autores Edgar Morin y Basarab Nicolescu sobre el concepto de la transdisciplinariedad y el paradigma del pensamiento complejo como prácticas educativas modernas para mejorar el proceso educativo.

\section{Objetivos específicos}

- Describir los conceptos de transdisciplinariedad y pensamiento complejo, desde los autores mencionados. 
- Determinar puntos comunes y divergentes entre ambas posturas epistémicas de los autores.

- Establecer algunos impactos positivos que devienen con la transdisciplinariedad.

\section{Desarrollo}

\section{La transdisciplinariedad de Basarab Nicolescu} y la epistemología del pensamiento complejo de Edgar Morin

Es importante comenzar señalando que esa dimensión epistémica del pensamiento complejo que postula Morin (2005) se basa en la transgresión de las fronteras disciplinares. Este autor es uno de los intelectuales que ha centrado buena parte de sus trabajos en la crítica al conocimiento fragmentado que imposibilita una comprensión de la realidad, la cual, de por sí, es cada vez más compleja.

Según el pensamiento de Morin, es justamente por ese aprendizaje fragmentario que se ha incurrido en un saber parcializado y doctrinario, donde cada disciplina o área del saber cree, para sí, tener la forma "correcta" o "adecuada" de aprehender y entender el mundo (Morin, 2002; Morin, 2005).

Para Morin (2005), la realidad es mucho más compleja. Por tanto, su comprensión debe darse desde otro punto de vista más complejo y global, el cual divide en tres niveles:

El primer nivel o base que se compone de las grandes teorías contemporáneas, las cuales son: la teoría de la información, la teoría general de sistemas y la teoría cibernética (Morin, 2005).
El segundo nivel que menciona Morin (2005) de ese mundo complejo tiene que ver con las teorías de la llamada autoorganización provenientes del campo de la biología y también incluye dentro de este nivel las teorías del campo de la cibernética.

El tercer nivel, Morin (2005) lo denomina el pensamiento complejo, al cual define como la posibilidad de pensar la existencia del ser humano, a partir de conglomerados teóricos anteriores desde el existencialismo, sobre todo retomando a Heidegger y a Husserl.

En esa línea, Morin (2005) traza su camino intelectual donde persigue romper la linealidad simple de la ciencia clásica, que todo lo ve unívoca y doctrinariamente, por un paradigma que contemple la complejidad so pena de la tendencia a la simplificación.

En palabras del propio Morin (2004): "la epistemología de la complejidad es una noción por explorar, a definir. La complejidad nos aparece, ante todo, efectivamente como irracionalidad, como incertidumbre, como angustia, como desorden" (p. 2). Como puede verse, desafortunadamente, existe una densidad que, a juicio de muchos detractores, no permite ver con claridad que lo que Morin está haciendo es construir una nueva racionalidad, tendiente a lo que él llama: un paradigma de complejidad o en términos más sencillos, un pensamiento complejo. En suma, se puede decir que esa complejidad, antes descrita a partir de Morin (2004), no está directamente relacionada con los asuntos exclusivos de las llamadas ciencias de la comple- 
jidad, sino que trata de abrir un nuevo horizonte conceptual que permita la emergencia y comprensión de un nuevo ser, superando la simplicidad de los esquemas de la ciencia clásica. En otras palabras, esa complejidad de Morin busca construir estrategias de pensamiento y acción, que posibiliten al ser humano enfrentar mejor el mundo en que vive, comprendiendo lo paradigmático del conocimiento (Morin, 2002; Morin, 2005; Osorio, 2012).

Partiendo de un mismo punto, aunque con enfoques y consideraciones diferentes, Nicolescu (1998) reconoce como principio de realidad, la complejidad del mundo, la interconectividad del mundo en red -que, en palabras de él mismo, resulta a veces enredado- y otros tantos factores que hacen el mundo y la realidad de los seres humanos muy compleja.

Nicolescu (1998) reconoce -al igual que lo hace Morin- que ese mundo complejo posee sin número de interconexiones que trascienden las fronteras clásicas de los fenómenos sociales y los naturales. Por tanto, concluye Nicolescu (1998) que no es posible seguir apegados al entendimiento de la realidad a partir del conocimiento fragmentado, porque la realidad es un todo complejo, que no debe comprenderse desde el aislamiento analítico de cada fenómeno por separado.

Lo anterior es otro punto coincidente entre el pensamiento de Morin y Nicolescu, ya que no solo parten de la realidad compleja, sino que también concuerdan -cada uno a su modo- en que, para entender mejor esa realidad, es necesaria una nueva racionalidad que contemple lo complejo y que, además, permita una nueva forma práctica, para analizar la realidad (Morin, 1998; Morin, 2004; Morin, 2005; Nicolescu et al., 1994; Nicolescu, 1998; Nicolescu, 1999).

Mientras que Morin (2002) habla de la transgresión de la disciplinariedad, como parte de ese paradigma complejo; Nicolescu (1999) plantea una transdisciplinariedad que permita integrar la complejidad del mundo en los conocimientos y prácticas. Algo así como una revolución ontológica, metodológica, epistemológica y espiritual del conocimiento humano (Osorio, 2012).

En ambos casos, existe, además, la convicción de que las universidades modernas constituyen la mejor forma y espacio de institucionalización de los saberes fragmentados que ambos combaten. Para Nicolescu (1998), si bien son útiles las razonables y profundas miradas disciplinares, son incompletas para la comprensión de la realidad. De allí que abogue por la búsqueda de ir más allá, traspasando esas fronteras de cada disciplina o área del saber específico, por medio de la transdisciplinariedad que hace posible un nuevo modo de articulación de los saberes.

En palabras del propio Nicolescu (1998): "la transdisciplinariedad consiste en el estudio de un objeto de una sola y misma disciplina, por varias disciplinas a la vez; el objeto en última instancia se verá enriquecido por la intersección de varias disciplinas" (p. 37). 
Encuentros y desencuentros de Edgar Morin y Basarab Nicolescu

Tal como fue señalado anteriormente, existen varios puntos comunes entre ambos autores que son objeto de reflexión en este ensayo. El primero de ellos es el reconocimiento de la realidad compleja, donde no solo los descubrimientos y avances en campos como la biología, la medicina, la informática, la inteligencia artificial, entre otros, han acentuado esa complejidad teórica y práctica. Además, coinciden en que, como resultante de ese escenario complicado, se crean y recrean de modo constante nuevas conexiones e interconexiones, contribuyendo todo ello a profundizar la complejidad ya existente.

A partir de lo anterior, ambos concuerdan que no es oportuno -hoy en día- apegarse al sistema clásico tradicional de enseñanza y aprendizaje lineal y muy mecánico, que se enseña por lo general en las universidades. Esto -afirman los dos- se ve reflejado en la enseñanza fragmentaria de las distintas disciplinas o saberes.

Por eso hacen su apuesta epistemológica -cada uno a su propio modo y estilo- para romper con esa unidimensionalidad; en el caso de Morin, lo llama la transgresión de las disciplinas como un nuevo modo de pensar y actuar (Morin, 1998; Morin, 2002; Morin, 2005), que posibilite la comunicación entre los distintos campos (el estrictamente científico y, también, sin dejar de lado al sujeto en su dimensión humana).

En eso también existe coincidencia con Nicolescu, ya que, si bien no habla de transgresión disciplinar, emplea el concepto de transdisci- plinariedad, pero son en el fondo muy similares uno del otro (Nicolescu et al., 1994, Nicolescu, 1998; Nicolescu, 1999). Porque para ambos, el punto por alcanzar reside en lograr una integración de distintas disciplinas o saberes, para su interpretación, sin caer en la disociación paradigmática que genera justamente el choque entre cada campo o área del saber.

Morin (2002) sostiene que es necesaria una nueva epistemología del pensamiento complejo, en la cual se considere y reflexione sobre lo que hay más allá del propio y estricto campo disciplinar, para que las disciplinas no se vuelvan automatizadas ni mecánicas.

Nicolescu, por su lado, parte del principio de que: "la naturaleza no puede ser conocida fuera de sus relaciones con el hombre, por ello la transdisciplinariedad no es una disciplina nueva, es algo que concierne a la relación entre las disciplinas y más allá de ellas" ( Nicolescu , 1996, citado en Hernández, Del Castillo y Castañeda, 2000, p. 15).

En ese sentido, puede verse que ambos autores, aunque con matices y énfasis algo distintos, comparten la necesidad de una integración de los saberes de áreas temáticas aparentemente dispares o distintas, para alcanzar la construcción de una visión holista que permita estudiar las conexiones que se dan entre ellas.

Quizás la mayor diferencia entre ambos radica en la forma (metodología) para alcanzar esa ruptura que lleve al holismo anhelado. Ya que, para Nicolescu (1996) citado en Osorio (2012), el estatuto metodológico de la transdisciplinariedad plantea el camino por medio de tres axiomas metodológicos: 
1. El axioma ontológico, que abarca la comprensión de los elementos de la naturaleza, los distintos niveles de realidad y los diferentes niveles de percepción (Osorio, 2012).

2. El axioma lógico, que según Nicolescu es el paso de un nivel de la realidad a otro, mediante la lógica interpretativa de un tercero (Osorio, 2012).

3. El axioma epistemológico, que remite a la estructura de la totalidad de niveles de realidad y de percepción, que son la base para la comprensión de la estructura compleja (Osorio, 2012).

Morin, por su parte, posee un abordaje metodológico algo distinto al de Nicolescu (Osorio, 2012). Ya que para Morin los aspectos básicos son:

1. El principio dialógico que, como su nombre lo indica, remite a generar un diálogo entre dos o más conceptos o nociones, que en apariencia son disímiles y opuestos. Tiende a unir posiciones contrarias, que son parte de la complejidad (Osorio, 2012).

2. El principio de recursión va ligado al principio anterior. Propiciando esfuerzos para que se generen consensos y productos concretos (una y otra vez) a partir de esos diálogos (Morin, 2005).

3. El principio hologramático sostiene que el sujeto es parte del todo y viceversa. Por tanto, existe en la práctica una conectividad intrínseca y, a veces, inconsciente en la acción (Morin, 2002).

\section{Impactos positivos del pensamiento complejo} y la transdisciplinariedad

Tal como se ha venido mencionando, el pensamiento complejo implica un asunto de cambio que conlleva -entre otras cosas- un proceso de ida y vuelta perenne entre las mal llamadas “certezas" y las “incertidumbres". Entre lo unitario y la totalidad. Entre lo divisible e indivisible. Es un proceso caórdico -para usar el concepto que se utiliza en la física cuántica- en donde se va configurando un orden dentro del propio caos.

Varios autores como Morin (1998), Torres (1998), Santos (2000) citado en Sobarzo (2001), Teubal (2008), Leff (2011), García (2016) y Estrada (2018) han venido sosteniendo que el cambio al paradigma complejo debe propiciar un aprendizaje constante desde un enfoque transdisciplinar. El dilema -por así decirlo- es cómo concreta su instrumentalización.

Hernández et al. (2000) sostienen que la implementación de la transdisciplinariedad en la educación significa importantes contribuciones al advenimiento de un nuevo tipo de educación, porque se centra en comprender las distintas conexiones de sentido que existen en la práctica, respecto de un mismo tema o fenómeno, pero desde múltiples aristas, enriqueciendo siempre con ello el proceso de aprendizaje.

En esa misma línea, Hernández et al. (2000) siguen señalando que la ruta crítica para instrumentalizar ese cambio transgresor -que tanto Morin como Nicolescu promulgan- es dando énfasis a cuatro pilares propios de la transdiscipli- 
nariedad: aprender a conocer, aprender a hacer, aprender a conciliar posiciones y aprender a ser. Otro aspecto positivo que la implementación del pensamiento complejo y la transdisciplinariedad tienen, es que, en estos tiempos modernos plagados de nuevas tecnologías de la información y comunicación, trabajo en redes, etc., facilitarían algo que resulta vital: que las personas tengan una mentalidad acorde a esa pluridimensionalidad, no solo en términos de los múltiples y muy diversos lenguajes, sino también la apertura mental y práctica hacia la gama enorme de distintas tecnologías que se encuentran hoy más que antes a la mano de las personas.

\section{Conclusiones}

Luego de todas las lecturas y consecuentes reflexiones de estas, queda claro que la ciencia sigue -pese a todo- con el ideal de la unificación de los saberes. Algo así como lo intentaron Platón, Hegel, Parsons, Einstein, entre otros. Generar un paradigma explicativo que contemple la totalidad.

Esto aplicado a la educación representa, al menos desde las dos perspectivas estudiadas en este caso, la pretensión como ideal de un holismo pedagógico que vaya más allá de lo estrictamente teórico, sino que también incluya los otros aspectos inherentes a la persona en tanto individuo (sentimientos, visiones de mundo, condicionamientos sociales, etc.).

Lo anterior para potenciar el surgimiento no solo de una nueva forma de entender y explicar el mundo, sino que se consiga un desarrollo global (integral) de las personas.
La clave está, según lo planteado por Morin y Nicolescu, en generar un diálogo permanente entre los distintos saberes, que tiendan a complementarse, con el fin de verse a sí mismas no como una isla totalitaria del saber, sino como un elemento más dentro del todo que, si se complementa con los otros elementos existentes, puede generar una sinergia importante que, a la postre, produzca acciones, ideas, así como prácticas educativas y de aprendizaje nuevas.

La actualidad del tema es innegable. El gran problema -por así decirlo- está en que, por mucho que se habla, sigue siendo eso, un ideal por alcanzar.

En buena parte, habría que reflexionar a fondo, luego, los diversos factores que no han posibilitado ese cambio de paradigma, si bien muy anhelado, no se logra concretar. Por lo pronto, baste señalar que, si los actores sociales involucrados en el proceso educativo (sector institucional, docentes, discentes, padres de familia, entre otros) siguen sin un compromiso claro hacia la concreción de este cambio de un paradigma tradicional a uno nuevo como el que se ha planteado en forma sucinta aquí, difícilmente pasará de verse como algo novedoso y deseable de alcanzar.

El intento aquí realizado responde a un acercamiento conceptual y de enfoque de la transdisciplinariedad, para que, con un conocimiento mayor, permita adentrarse ulteriormente en la necesaria instrumentalización de ese cambio paradigmático. Para eso, sin duda, se requiere de compromiso. 


\section{Referencias}

Enwistle, H. (2011). Class, Culture and Education. Routledge Library Editions.

Escudero, A. (2011). Ciudadanía y educación para la ciudadanía [Tesis doctoral, Universidad de Castilla-La Mancha, España]. Repositorio RUIdeRA. https://ruidera.uclm.es/xmlui/handle/10578/2956

Estrada, A. (2018). El pensamiento complejo y el buen vivir como epístemes emergentes para comprender la formación docente desde la diversidad. Revista Arbitrada Del Centro de Investigación y Estudios Gerenciales, 34(2), 221-235. http://www.grupocieg.org/archivos_revista/ Ed.34\%20(221-235)\%20Alex\%20Estrada_articulo_id427.pdf

Fedorov, A. (2008). Foro virtual como una estrategia metodológica para el desarrollo del pensamiento crítico en la universidad. Tecnología en Marcha, 21(3), 102-113. https://revistas.tec. ac.cr/index.php/tec_marcha/article/view/189

García, J. (2016). Una práctica religante de saberes fragmentados en el contexto de un siglo signado por la incertidumbre y la complejidad. En J.M. González (Ed.), Transdisciplinariedad en la educación (pp. 100-114). https://www.researchgate.net/profile/Juan_Miguel_Gonzalez_Velasco/publication/341056581_TRANSDISCIPLINARIEDAD_EN_LA_EDUCACION_DOCENCIA_ESCUELA_Y_AULA/links/5eab3f26299bf18b958a7147/TRANSDISCIPLINARIEDAD-EN-LA-EDUCACION-DOCENCIA-ESCUELA-Y-AULA.pdf\#page=102

Hernández, L., Del Castillo, A. y Castañeda, E. (2000). La transdisciplinariedad, una acción prioritaria para la educación superior a comienzos del Tercer Milenio. Revista Pedagogía Universitaria, 5(1), 13-18.

Leff, E. (2006). Aventuras de la epistemología ambiental: de la articulación de ciencias al diálogo de saberes. Siglo XXI.

Morin, E. (1998). Articular los saberes. ¿Qué saberes enseñar en las escuelas? Ediciones Universidad del Salvador. https:/es.scribd.com/document/112914020/Morin-Edgar-Articular-los-saberes-textos-escogidos-Que-saberes-ensenar-en-las-escuelas

Morin, E. (2002). La cabeza bien puesta. Nueva Visión. http://ipcem.net/wp-content/ uploads/2014/08/La_cabeza_bien_puesta.pdf

Morin, E. (2005). Introducción al pensamiento complejo. Gedisa. http://cursoenlineasincostoedgarmorin.org/images/descargables/Morin_Introduccion_al_pensamiento_complejo.pdf 
Nicolescu, B., Morin, E., Anes, J., Astier, A., Bastien, J., Berger, R., Bianchi, F. ... Viera, A. (1994). Carta de la transdisciplinariedad. Convento de Arrábida, Portugal. https://www.filosofia.org/cod/c1994tra.htm

Nicolescu, B. (1998). La transdisciplinariedad. Manifiesto. 7 Saberes. https://basarab-nicolescu. fr/BOOKS/Manifeste_Espagnol_Mexique.pdf

Nicolescu, B. (1999). La necesidad de la transdisciplinariedad en la educación superior. Trans-pasando fronteras, 3, 23-30. https://doi.org/10.18046/retf.i4.1779

Osorio, S. (2012). El pensamiento complejo y la transdisciplinariedad: fenómenos emergentes de una nueva racionalidad. Revista Facultad de Ciencias Económicas: investigación y reflexión, $X X(1)$. http://www.scielo.org.co/pdf/rfce/v20n1/v20n1a16.pdf

Sobarzo, O. (2001). Por uma outra globalização: do pensamento único à consciência universal. EURE (Santiago), 27(81), 129-131. http://dx.doi.org/10.4067/S0250-71612001008100008

Teubal, M. (2008). Globalización y nueva ruralidad en América Latina. CLACSO. http://biblioteca.clacso.edu.ar/clacso/gt/20100929011903/4teubal.pdf

Torres, J. (1998). Globalización e interdisciplinariedad: el curriculum integrado (3ra ed.). Ediciones Morata. http://www.terras.edu.ar/biblioteca/1/CRRM_Torres_Unidad_2.pdf 